\title{
Protocolo de scoping review: auriculoterapia em crianças e adolescentes no âmbito
}

\section{da Atenção à Saúde}

Scoping review protocol: auriculotherapy in children and adolescents in the context of Health Care

Protocolo de revisión de alcance: auriculoterapia em niños y adolescentes en el contexto de la Atentión Sanitaria

Recebido: 05/12/2021 | Revisado: 10/12/2021 | Aceito: 13/12/2021 | Publicado: 21/12/2021

Karla Adriana Ferreira Beckman ORCID: https://orcid.org/0000-0003-2792-0758

Universidade Católica do Salvador, Brasil E-mail: dra.karlabeckman@gmail.com

Marimeire Morais da Conceição ORCID: https://orcid.org/0000-0002-9568-6468 Universidade Federal da Bahia, Brasil E-mail: enfufba2002@yahoo.com.br

Denízia Maria Barberino Xavier Santos ORCID: https://orcid.org/0000-0002-5186-0647

Universidade Católica do Salvador, Brasil Universidade Federal da Bahia, Brasil E-mail: denizia2@gmail.com

Márcia Aparecida Ferreira de Oliveira ORCID: https://orcid.org/0000-0002-1069-8700 Universidade de São Paulo, Brasil Universidade Federal de Santa Maria, Brasil E-mail: marciaap@usp.br Ana Maria Fernandes Pitta

ORCID: https://orcid.org/0000-0003-2558-5757 Universidade Católica do Salvador, Brasil E-mail: ana.maria.pitta@gmail.com

\begin{abstract}
Resumo
O objetivo deste estudo é mapear e analisar a produção científica acerca da auriculoterapia para crianças e adolescentes no âmbito da atenção à saúde. Para tanto, foi utilizada a estratégia PCC: (P) Participantes - Crianças e Adolescentes entre 6 e 12 anos (OMS); (C) conceito: auriculoterapia; e (C) Contexto: atenção integral à saúde, como recomendam estudiosos (Aromataris \& Munn, 2020) . Que originou a seguinte questão: Quais as evidências na literatura científica sobre auriculoterapia para crianças e adolescentes no âmbito da atenção integral à saúde? Esta revisão de escopo considerará estudos originais, realizados exclusivamente com seres humanos, crianças com idade entre 6 e 12 anos incompletos, publicados na íntegra em periódicos nacionais e internacionais, gratuitos ou não, com abordagem qualitativa e/ou quantitativa, nos idiomas português, inglês e espanhol. Os estudos deverão abordar o uso da auriculoterapia na atenção à saúde. Assim, serão considerados os estudos com as variações de recursos da auriculoterapia (sementes de vaccaria, esferas magnéticas e cristais). As fontes indexadas que serão utilizadas para a revisão de escopo são: Medical Literature Anallysis and Retrieval System online (MEDLINE); Literatura LatinoAmericana e do Caribe em Ciências da Saúde (LILACS); Scientific Electronic Library online (SCIELO); as bases de dados Cochrane Database of Systematic Reviews (CDCR), a Bibliografia Brasileira de Odontologia (BBO), bem como a Biblioteca Virtual em Saúde em Medicinas Tradicionais, Complementar e Integrativa (BVS MTCI), Biblioteca Virtual em Saúde do Adolescente (ADOLEC) e Physiotherapy Evidence Database (PEDro). Para pesquisar a literatura cinzenta, será consultada a Coordenação de Aperfeiçoamento de Pessoal de Nível Superior (CAPES). As buscas nestas fontes indexadas serão realizadas no período entre fevereiro e março de 2022. Espera-se com esta revisão contribuir com o estado da arte acerca da produção e implementação de Práticas Integrativas Complementares.
\end{abstract}

Palavras-chave: Adolescente; Atenção Integral à Saúde; Auriculoterapia; Criança.

\section{Abstract}

The objective of this study is to map and analyze the scientific production about auriculotherapy for children and adolescents in the context of health care. For that, the PCC strategy was used: (P) Participants - Children and Adolescents between 6 and 12 years old (WHO); (C) concept: auriculotherapy; and (C) Context: comprehensive health care, as recommended by scholars (Aromataris \& Munn, 2020). Which gave rise to the following question: What is the evidence in the scientific literature on auriculotherapy for children and adolescents in the context of 
comprehensive health care? This scope review will consider original studies, carried out exclusively with human beings, children aged between 6 and 12 years old, published in full in national and international journals, free or not, with a qualitative and/or quantitative approach, in Portuguese, English and Spanish. Studies should address the use of auriculotherapy in health care. Thus, studies with variations in auriculotherapy resources (vaccaria seeds, magnetic spheres and crystals) will be considered. The indexed sources that will be used for the scope review are: Online Medical Literature Analysis and Retrieval System (MEDLINE); Latin American and Caribbean Literature on Health Sciences (LILACS); Scientific Electronic Library online (SCIELO); the Cochrane Database of Systematic Reviews (CDCR), the Brazilian Dentistry Bibliography (BBO), as well as the Virtual Health Library in Traditional, Complementary and Integrative Medicines (BVS MTCI), Adolescent Health Virtual Library (ADOLEC) and Physiotherapy Evidence Database (PEDro). To search the gray literature, the Coordination for the Improvement of Higher Education Personnel (CAPES) will be consulted. Searches in these indexed sources will be carried out between february and march 2022. It is expected that this review will contribute to the state of the art regarding the production and implementation of Complementary Integrative Practices in Health (PICS).

Keywords: Adolescent; Comprehensive Health Care; Auriculotherapy; Child.

\section{Resumen}

El objetivo de este estudio es mapear y analizar la producción científica sobre la auriculoterapia para niños y adolescentes en el contexto de la atención de la salud. Para ello se utilizó la estrategia de CCP: (P) Participantes Niños y Adolescentes entre 6 y 12 años (OMS); (C) concepto: auriculoterapia; y (C) Contexto: atención integral de salud, según lo recomendado por los académicos (Aromataris \& Munn, 2020). Lo que dio lugar a la siguiente pregunta: ¿Cuál es la evidencia en la literatura científica sobre la auriculoterapia para niños y adolescentes en el contexto de la atención integral de salud? Esta revisão de escopo considerará estudos originais, realizados exclusivamente com seres humanos, crianças con idade entre 6 e 12 anos incompletos, publicados na íntegra em periódicos nacionais e internacionais, gratuitos ou não, com abordagem qualitativa e/ou quantitativa, nos idiomas português, inglês y español. Los estudios deben abordar el uso de la auriculoterapia en el cuidado de la salud. Así, se considerarán estudios con variaciones en los recursos de auriculoterapia (semillas de vaccaria, esferas magnéticas y cristales) Las fuentes indexadas que se utilizarán para la revisión del alcance son: Sistema de Análisis y Recuperación de Literatura Médica en Línea (MEDLINE); Literatura Latinoamericana y del Caribe en Ciencias de la Salud (LILACS); Biblioteca Electrónica Científica en línea (SCIELO); la Base de Datos Cochrane de Revisiones Sistemáticas (CDCR), la Bibliografía Brasileña de Odontología (BBO), así como la Biblioteca Virtual de Salud en Medicamentos Tradicionales, Complementarios e Integrativos (BVS MTCI), la Biblioteca Virtual de Salud Adolescente (ADOLEC) y la Base de Datos de Evidencia de Fisioterapia (PEDro ). Para la búsqueda de literatura gris se consultará a la Coordinación de Perfeccionamiento del Personal de Educación Superior (CAPES). Las búsquedas en estas fuentes indexadas se llevarán a cabo entre febrero y marzo de 2022. Se espera que esta revisión contribuya al estado del arte en la producción e implementación de Prácticas Integrativas Complementarias en Salud (PICS).

Palabras clave: Adolescente; Atención Integral de Salud; Auriculoterapia; Niño.

\section{Introdução}

A auriculoterapia vem do grego "terapia" que sugere curar e a palavra "aurícula" vem do latim que significa orelha. Esta prática consiste no tratamento de diferentes doenças através da orelha e se apresenta como um ramo da acupuntura que pode usar agulhas chinesas e/ou sementes. Paul Nogier foi o primeiro a criar o mapa detalhado do pavilhão auricular em que destacava que cada indivíduo possui características próprias em suas orelhas quanto à forma, largura, comprimento e posição em relação à cabeça (Esteriz Mesidoro, et al., 2020).

Para (Corrêa, et al., 2020), a auriculoterapia é considerada prática integrativa e complementar em saúde na qual o mecanismo de ação da intervenção é explicado pela função somatotrópica, isto é, devido a correspondência ponto a ponto de uma área do corpo com um ponto específico no sistema nervoso central. Além disso, relaciona-se com os fundamentos da Medicina Tradicional Chinesa (MTC), associando o corpo com os doze meridianos (usados na acupuntura) que chegam às suas ramificações de forma direta ou indireta até o pavilhão auricular.

Os meridianos yang (intestino grosso, intestino delgado, vesícula biliar, triplo aquecedor, estômago e bexiga) passam ao redor orelha ligando-se de maneira direta, os yins (pulmão, coração, pericárdio, fígado, baço/pâncreas e rins) se combinam a orelha por meio de ramificações, de modo que os 12 meridianos chegam igualmente até a orelha. Assim, quando algum meridiano tem seu fluxo obstruído no corpo, aparecem pontos dolorosos na orelha, como uma reação reflexa do local 
obstruído. Além disso, as funções dos órgãos e vísceras (Zang Fu) descritas na MTC podem ser estimuladas através dos pontos auriculares (Fonseca, 2011).

Neste sentido, utilizar a prática da auriculoterapia como estratégia de cuidado na saúde da criança e de adolescente favorecerá a prevenção de doenças, a promoção e a recuperação da saúde (Brasil, 2012; Brasil, 2015). Estudos realizados sugerem que o uso da auriculoterapia é uma tecnologia efetiva e segura, pois os efeitos adversos são substancialmente menores quando comparados ao uso de medicamentos. Além disso, também foi verificado que uso da auriculoterapia para crianças e adolescentes com obesidade, miopia e transtorno de atenção e hiperatividade (TDAH) também é seguro (Cha \& Park, 2019; Nielsen \& Gereau, 2020; Gao, et al., 2020; Binesh, et al., 2020).

Entendendo a auriculoterapia como uma prática com ênfase na escuta acolhedora é possível estabelecer vínculo terapêutico e integração do indivíduo com o meio ambiente e com a sociedade em que vive. Tais interações integram ações que, em conjunto, são responsáveis por viabilizar a atenção integral e humanizada durante o acompanhamento do desenvolvimento saudável de crianças e adolescentes (Brasil, 2012; Brasil, 2015).

A exemplo, diversas Políticas e Programas nacionais e internacionais recomendam a aplicação da medicina tradicional chinesa como meio de promover o fortalecimento do vínculo mãe-bebê usando massagens e tratamentos de doenças comuns na infância. Isto ocorre porque nesses procedimentos, é possível compreender os diferentes processos vitais e a gênese das doenças que envolvem a predisposição do sujeito e as modalidades de interações socioculturais e com o ambiente (Brasil, 2012).

Assim, compreendendo a importância das práticas integrativas e complementares em saúde na melhora da qualidade de vida do sujeito. Portanto, levando em consideração que o uso da auriculoterapia permite cuidar de crianças e adolescentes usando material de baixo custo, apresentamos a seguinte questão problema: quais as evidências na literatura científica sobre auriculoterapia para crianças e adolescentes no âmbito da atenção integral à saúde? Para responder esta pergunta, objetivamos examinar e mapear na literatura científica o que se tem produzido acerca da auriculoterapia para crianças e adolescentes no âmbito da atenção à saúde.

O presente estudo tem sua relevância por contribuir com o estado da arte acerca da produção e implementação de Práticas Integrativas Complementares em Saúde (PICS), mais especificamente a Auriculoterapia, em crianças e adolescentes. Logo, favorece o incremento do uso dessas estratégias neste público, ampliando a acessibilidade e continuidade do cuidado, bem como favorece a diminuição da medicalização que é considerada voltada para uma abordagem biomédica, caracterizada como invasiva, restrita aos sintomas e não aos aspectos biopsicossociais.

\subsection{Objetivos}

Mapear e analisar a produção científica acerca da auriculoterapia para crianças e adolescentes no âmbito da atenção à saúde.

Outros objetivos são:

1. Identificar os principais agravos e doenças que são tratadas pela auriculoterapia em crianças e adolescentes no âmbito da atenção à saúde;

2. Identificar os agravos ou condições de saúde em que a auriculoterapia é utilizada em crianças e adolescentes

3. Identificar os recursos mais utilizados na auriculoterapia em crianças e adolescentes

4. Verificar quais métodos de pesquisas são mais utilizados para abordar a temática da auriculoterapia em crianças e adolescentes

5. Identificar os participantes mais comuns em pesquisas envolvendo a auriculoterapia em crianças e adolescentes

6. Descrever as lacunas apontadas por estudos que abordam a auriculoterapia em crianças e adolescentes 
7. Apontar a qual a área da saúde que mais investiga o uso da auriculoterapia em crianças e adolescentes

8. Verificar os benefícios apontados pelos estudos sobre o auriculoterapia em crianças e adolescentes

\section{Metodologia}

\subsection{Tipo de Estudo}

Trata-se de uma revisão de escopo, no modelo Joanna Briggs Institute (JBI), com o objetivo de ampliar as fontes de pesquisa nesta determinada área, usando não somente os artigos científicos, mas também teses, monografias e outras produções científicas, com isso, acrescentar dados da investigação e identificar possíveis lacunas nas pesquisas existentes, que possibilitará uma síntese das evidências produzidas acerca da temática escolhida. De maneira geral, a revisão de escopo tem como objetivo mapear os estudos no campo de interesse abordando tópicos mais amplos em que diferentes desenhos de estudos (estudos experimentais, não experimentais, dados da literatura empírica e teórica) poderão ser utilizados na pesquisa, não havendo, portanto, a preocupação para evidenciar o melhor tipo de estudo, mas sim apresentar as evidências produzidas (Cordeiro \& Soares, 2019; Aromataris \& Munn, 2020).

Além disso, a escolha por este tipo de revisão deu-se também com intuito de buscar na literatura o reconhecimento dos principais conceitos/definições sobre o tema a ser pesquisado. Neste tocante, é possível identificar na literatura não somente o que já foi explorado, como também, identificar lacunas no campo de interesse para posteriormente recomendações de pesquisas futuras (Arkey \& O'Malley, 2005).

De acordo (Aromataris \& Munn, 2020) foi realizada uma revisão de escopo sobre a temática "revisão de escopo" e foi descoberto que os três motivos mais comuns para conduzir uma revisão de escopo eram explorar a amplitude ou extensão da literatura, mapear e resumir as evidências e informar pesquisas futuras. Dessa maneira, entende-se que a escolha por este método oportunizará examinar a extensão e natureza das produções e/ou esclarecer conceitos que fundamentam o uso auriculoterapia como estratégia de cuidado na saúde integral de crianças e adolescentes.

O processo da revisão de escopo seguirá nove etapas: 1-Desenvolver e registrar o protocolo; 2-Submissão do Protocolo; 3-Perguntas e Objetivos; 4-Desenvolver critérios de inclusão e exclusão; 5- Descrever a Triagem e Seleção das Evidências; 6-Buscar as evidências em três etapas (títulos, resumo e leitura do texto na íntegra); 7-Selecionar os artigos; 8Extração dos dados; 9-Mapear e Resumir os dados; conforme a metodologia Joanna Briggs Institute (JBI) (Peters, et al., 2020).

Sendo assim, no presente protocolo, onde pré-define-se os objetivos, métodos e relatórios da revisão permitindo assim a transparência do processo ${ }^{11}$. Dessa forma, para auxiliar na construção da pergunta foi utilizada a estratégia PCC à saber: $(\mathrm{P})$ Participantes - Crianças e Adolescentes entre 6 e 12 anos (OMS); (C) conceito: auriculoterapia; e (C) Contexto: atenção integral à saúde, como recomendam estudiosos (Aromataris \& Munn, 2020).

\subsection{Pergunta de Pesquisa}

Quais as evidências na literatura científica sobre auriculoterapia para crianças e adolescentes no âmbito da atenção integral à saúde?

Outras questões de pesquisa a serem investigadas são:

1. Quais os agravos ou condições de saúde em que a auriculoterapia é utilizada em crianças e adolescentes?

2. Quais os recursos mais utilizados na auriculoterapia em crianças e adolescentes?

3. Quais métodos de pesquisas são mais utilizados para abordar a temática da auriculoterapia em crianças e adolescentes?

4. Quais são os participantes mais comuns em pesquisas envolvendo a auriculoterapia em crianças e adolescentes?

5.Quais as lacunas apontadas por estudos que abordam a auriculoterapia em crianças e adolescentes?

6. Qual a área da saúde que mais investiga o uso da auriculoterapia em crianças e adolescentes?

7. Quais os benefícios apontados pelos estudos sobre o auriculoterapia em crianças e adolescentes? 


\subsection{Critérios de Inclusão}

Para critérios de inclusão serão considerados editoriais, estudos originais, recomendações, reflexões, publicados na íntegra, cujos participantes sejam seres humanos, ou seja, crianças e adolescentes com idade entre seis e 12 anos incompletos, publicados na íntegra em periódicos nacionais e internacionais, gratuitos, com abordagem qualitativa e/ou quantitativa, e que fale sobre o uso da auriculoterapia na atenção à saúde. Assim, serão considerados os estudos com as variações de recursos da auriculoterapia (sementes de vaccaria, esferas magnéticas e cristais). Serão incluídos os textos nas modalidades em inglês, português e espanhol, considerando a limitação vocabular das autoras com relação a outros idiomas.

\subsection{Critérios de Exclusão}

Serão excluídos artigos duplicados, revisões, estudos que abordem a combinação da auriculoterapia com outra técnica terapêutica e que usem métodos de estimulação auricular (eletroestimulação, laser).

\subsection{Fontes de Coletas dos Dados}

Será realizada uma busca com os descritores (criança, adolescente, auriculoterapia, acupressão e saúde), de acordo com os descritores em saúde (DECS) e MESH nos idiomas inglês, português e espanhol, intercalados com os operadores boleanos AND e OR.

As bases de dados que serão utilizadas para a revisão de escopo: Medical Literature Anallysis and Retrieval System online (MEDLINE); Literatura Latino-Americana e do Caribe em Ciências da Saúde (LILACS); Scientific Electronic Library online (SCIELO); as bases de dados Cochrane Database of Systematic Reviews (CDCR), a Bibliografia Brasileira de Odontologia (BBO), bem como a Biblioteca Virtual em Saúde em Medicinas Tradicionais, Complementar e Integrativa (BVS MTCI), Biblioteca Virtual em Saúde do Adolescente (ADOLEC) e Physiotherapy Evidence Database (PEDro). Para pesquisar a literatura cinzenta, será consultada a Coordenação de Aperfeiçoamento de Pessoal de Nível Superior (CAPES).

\subsection{Período da coleta de dados}

As buscas nestas fontes indexadas serão realizadas no período entre fevereiro e março de 2022.

\subsection{Seleção dos Estudos}

Os estudos serão selecionados após a leitura do título, resumo e leitura na íntegra. Para facilitar este processo, os estudos serão agrupados e carregados no programa Rayyan, um aplicativo gratuito para web e celular que foi desenvolvido especificamente para otimizar a triagem inicial de resumos e títulos (Ouzzani, et al., 2016).

A seleção dos dados seguirá o fluxograma adaptado Preferred Reporting Items for Systematic reviews and MetaAnalyses extension for. Scoping Reviews, de nome PRISMA-ScR, recomendado por estudiosos (Trico, et al., 2018).

\subsection{Coleta e extração de dados}

Após a seleção dos artigos serão extraídos: título, autores e o respectivo campo de pesquisa na saúde (medicina, enfermagem, fisioterapia, psicologia, terapia ocupacional entre outras), ano de publicação, revista responsável pela publicação, palavras chaves e descritores utilizados. Sobre a metodologia: tipo de estudo, técnica de coleta de dados. 
Em caso de pesquisa de campo, serão extraídos: local onde ocorreu o estudo (cidade, estado e país); níveis de complexidade de atenção à saúde (atenção básica, ambulatorial, domiciliar ou hospitalar), agravo ou condição de saúde em que foi aplicada a auriculoterapia.

Quanto aos participantes serão investigadas: profissional: quantidade, idade, sexo, religião, raça/cor, área da saúde e tempo de atuação profissional; familiares: quantidade, idade, sexo, religião, raça/cor e vínculo de parentesco com a criança /adolescente. Se o estudo for realizado com crianças e adolescente será identificado: quantidade, idade, sexo, religião e raça/cor.

Sobre a auriculoterapia serão relacionados os seguintes dados: recursos utilizados (semente de vaccaria, cristais e esferas magnéticas), tempo de tratamento, possíveis dificuldades relatadas pelos autores e resultados alcançados. Também serão relacionadas às lacunas apontadas pelos estudos e as sugestões de realização de novos estudos.

\subsection{Análise dos Dados}

Os dados extraídos dos textos selecionados serão analisados e apresentados mediante a utilização de frequências simples e absolutas. Também, os resultados dos estudos serão lidos de forma aprofundada a fim de obter síntese dos resultados mais frequentes.

Posteriormente, as sínteses das evidências desses dados serão expressas em dados numéricos, quadros, gráficos, fluxogramas, entre outros. Esta análise de dados será finalizada com a síntese narrativa, como sugerem especialistas (Cordeiro \& Soares, 2019).

\section{Considerações Finais}

A produção deste protocolo possibilitará a realização de uma revisão de escopo embasa nos princípios estabelecidos pelo Joanna Briggs Institute (JBI) e, a partir dela, espera-se contribuir com o estado da arte acerca da produção e implementação de Práticas Integrativas Complementares em Saúde (PICS), por meio do uso da auriculoterapia em crianças e adolescentes no âmbito da atenção à saúde.

\section{Referências}

Arkey, H., \& O'Malley, L. (2005). Scoping Studies: Towards a Methodological Framework. International Journal of Social Research Methodology: Theory \& Practice, 8(1), 19-32. doi:10.1080/1364557032000119616.

Aromataris, E. \& Munn, Z. (2020). JBI Manual for Evidence Synthesis. JBI. doi:10.46658/JBIMES-20-01

Correa H.P., Moura C.C., Azevedo C., Bernardes, M.F.V.G., Mata, L.R.F.P., Chianca, T,C,M,.(2020). Effects of auriculotherapy on stress, anxiety and depression in adults and older adults: a systematic review. Revista da Escola de Enfermagem de USP. 54:e03626. doi:10.1590/S1980-220X2019006703626

Brasil (2012). Ministério da Saúde. Secretaria de Atenção à Saúde. Departamento de Atenção Básica. Saúde da criança: crescimento e desenvolvimento. Brasília: Ministério da Saúde, 1-272.

Brasil (2015). Ministério da Saúde. Secretaria de Atenção à Saúde. Departamento de Atenção Básica. Política Nacional de Práticas Integrativas e Complementares no SUS: atitude de ampliação de acesso. 2. ed. Brasília: Ministério da Saúde, 1-95.

Binesh, M., Daghighi, M.R., Shirazi, E., Oleson, T., Hashem-Dabagjian, F. (2020). Comparison of Auricular Therapy with Sham in Children with Attention Deficit/Hyperactivity Disorder: A Randomized Controlled Trial. J Altern Complement Med. Jun; 26(6), 515-520. doi:10.1089/acm.2019.0477.

Cordeiro, L. \& Soares, C. B.(2019). Revisão de escopo: potencialidades para a síntese de metodologias utilizadas em pesquisa primária qualitativa. Boletim do Instituto de Saúde - BIS, São Paulo, 20, (2), 37-43. https://docs.bvsalud.org/biblioref/2019/10/1021863/bis-v20n2-sintese-de-evidencias-qualitativas-37-43.pdf.

Cha, H.S., Park, H.( 2019). Efeitos da acupressão auricular na obesidade em adolescentes. Complement Ther Clin Pract. Maio, (35), 316-322. doi: 10.1016 / j.ctcp.2019.03.014.

Esteriz Mesidoro, N., Taboada Torres, A., Aponte González, M., \& Esteriz Mesidoro, Y. (2020). Auriculoterapia en pacientes con queratitis punteada superficial. MEDISAN, 24(3), 396-405. http://scielo.sld.cu/scielo.php?script=sci_arttext\&pid=S1029-30192020000300396\&lng=es\&tlng=es.

Fonseca, W.P. (2011). Acupuntura Auricular Chinesa. São Paulo: Andreoli. 
Research, Society and Development, v. 10, n17, e77101724368, 2021

(CC BY 4.0) | ISSN 2525-3409 | DOI: http://dx.doi.org/10.33448/rsd-v10i17.24368

Gao, H., Zhang, L., Liu, J. (2020). Auricular acupressure for myopia in children and adolescents: A systematic review. Complement Ther Clin Pract. doi: 10.1016/j.ctcp.2019.101067.

Nielsen, A. Gereau, S., Tick, H. (2020). Risks and Safety of Extended Auricular Therapy: A Review of Reviews and Case Reports of Adverse Events. Pain Med. Jun 1; 21, (6), 1276-1293. doi: 10.1093/pm/pnz379.

Peters, M., Marnie, C., Tricco, A. C., Pollock, D., Munn, Z., Alexander, L., McInerney, P., Godfrey, C. M., \& Khalil, H. (2020). Updated methodological guidance for the conduct of scoping reviews. JBI evidence synthesis, 18(10), 2119-2126. doi: 10.11124 / JBIES-20-00167

Ouzzani, M., Hammady, H., Fedorowicz, Z., Elmagarmid, A. (2106). Rayyan - a web and mobile app for systematic reviews. Syst Rev 5, 210. doi.org/10.1186/s13643-016-0384-4.

Tricco, A. C., Lillie, E., Zarin, W., O'Brien, K. K., Colquhoun, H., Levac, D., Moher, D., Peters, M. D., Horsley, T., Weeks, L., \& Hempel, S., (2018).

PRISMA extension for scoping reviews (PRISMA-ScR): checklist and explanation. Annals of internal medicine, 169(7), 467-473. doi: 10.7326 / M18-0850 